\title{
Paraplegia
}

\section{Experience with a Spinal Cord Unit in Brasilia, Brazil}

M. Masini MD, N. G. F. Neto, MD, E. G. C. Neves, MD

Hospital for Locomotor System Disease, SARAH, Brasilia 70.330, Brazil.

\section{Summary}

We have analysed our experiences in a Spinal Cord Unit in Brasilia, Brazil in the last 5 years (1982-1986). There were 738 outpatients and 243 inpatients. One hundred and six patients were operated on by the neurosurgical staff, and the indications for operation are discussed. Some patients showed neurological recovery with surgery and some without surgery. Patients with a complete neurological deficit on admission did not show any improvement, and those with a severe but incomplete deficit showed improvement with surgery $(18 \%)$, which was better than those without an operation. We conclude that a surgical procedure seems to be indicated in those with a comminuted, or with an unstable fracture, if there is an incomplete neurological deficit with an unreduced spinal deformity. There was a possibility of recovery of some nerve root function. The goal of surgical treatment is to restore and maintain the anatomical relationships between the spinal canal, spinal cord and nerve roots with the simplest possible surgical procedure, returning the patient as soon as possible to his functional capacity and social life.

Key words: Spinal Cord Unit; Brasilia; Brazil.

\section{Introduction}

Brazil is a developing country with more than 130 million inhabitants, and has to import part of its technological advances from other countries. As technology develops rapidly, we have to equate it to our needs and find space for our own developments. There has been a state medical system since 1940. There are few centres for rehabilitation of injured people. The three most important centres are located in Sao Paulo, Rio de Janeiro and Brasilia. The Hospital for Locomotor System Diseases, SARAH was founded in 1962 and expanded in 1982 to 300 beds, of which 100 are in the Spinal Unit (Fig. 1) It has intensive care, acute care, rehabilitation and social integration sections, and receives patients 


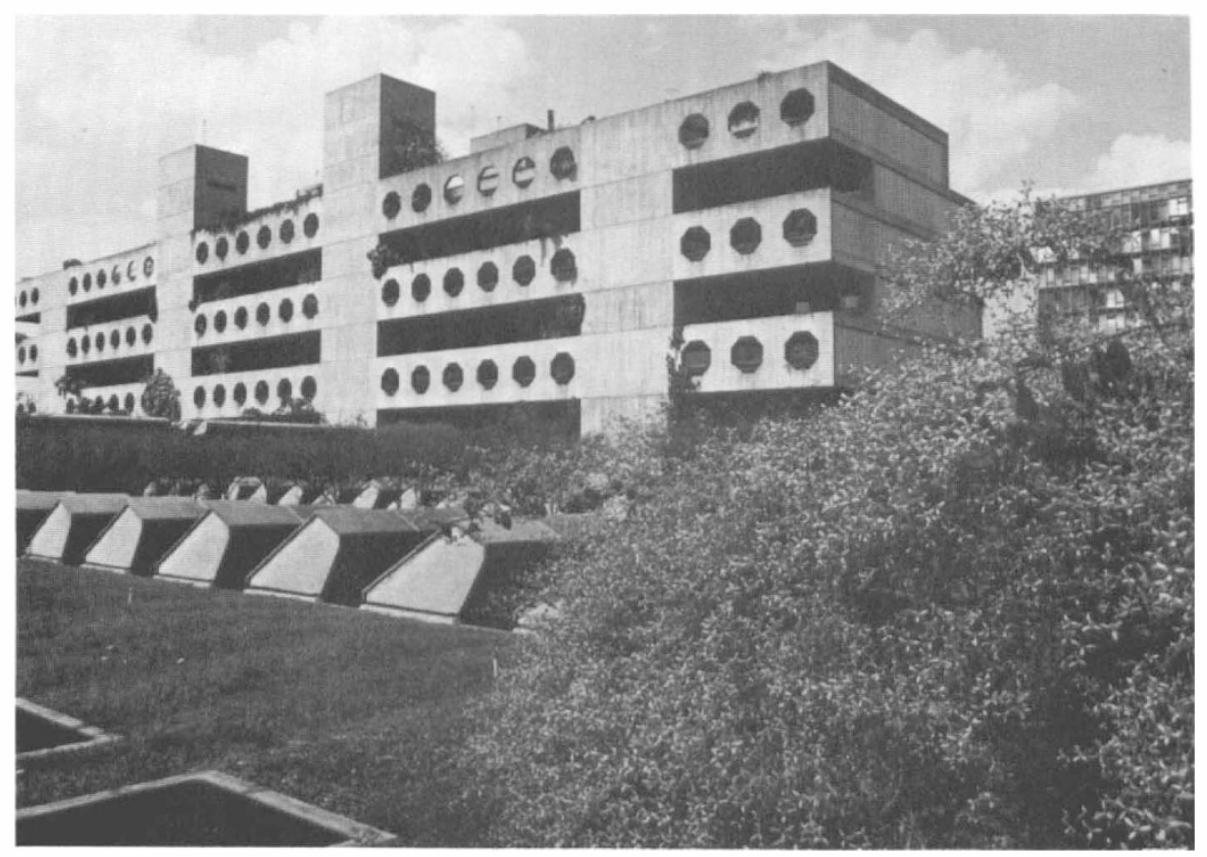

Figure 1 External panoramic view of the hospital for Locomotor System Disease.

from the North, Northeast and Central regions of Brazil which have a population of about 40 million people. The Unit is headed by physiatrists, and has neurosurgeons, orthopaedic surgeons, plastic surgeons, psychiatrists, physical therapists, nurses, rehabilitation specialists and social assistants. There is a rehabilitation gymnasium and the Equiphos which was specially created to design and manufacture hospital equipment appropriate to our country.

During the last 5 years (1982-86), 738 patients were treated in the outpatient clinic, all with a severe spinal cord injury; 243 patients were treated in the ward and of these 106 were operated on by the neurosurgical staff. This paper analyses the first 105 cases and compares the treatments, with special attention to our protocol.

\section{Clinical material and treatment}

The patients were admitted for treatment in the acute phase and submitted to a clinical, electrophysiological, and radiological evaluation. Based on the following protocol we decided on surgical or non-surgical treatment for the spinal cord injury (Green, 1981; Harris, 1978; Holdsworth, 1975; Kobrine, 1975; Masini and Walters, 1986).

The following variables were compared: the degree of the lesion; level; initial treatment; dependency on admission and on discharge; locomotion on admission and on discharge; complications on admission and during the hospital stay; bowel and bladder functions on admission and on discharge. 
To compare the severity of the lesion on admission and discharge we used Frankel's neurological classification (Frankel et al., 1962).

\section{Protocol}

Group 1: patients with neurological deficit, with no extrinsic pressure on the spinal cord or roots, as shown by myelography or computerised tomography and with spinal column stability. Treatment: intensive care unit for 10 days, followed by rehabilitation.

Group 2: patients with neurological deficit, with no extrinsic pressure on the spinal cord or roots as shown by myelography or computerised tomography and with spinal column instability. Treatment: intensive care unit for 10 days, and stabilisation of the spinal column, if surgical treatment is necessary, the surgery could be done between the tenth and the twenty first days, post-injury.

Group 3: patients with neurological deficit and myelography or computerised tomography findings of extrinsic compression of the spinal cord or roots, whose nervous system was neither decompressed by traction, nor by mobilisation. Treatment: surgery in the first 24 hours to decompress the neural structures and stabilisation of the vertebral column.

Group 4: patients with a neurological deficit due to a gunshot wound or from a knife in which there is a foreign body in the spinal canal or a CSF leak. Treatment: immediate surgery and closure of the CSF leak.

\section{Analysis}

Of 448 patients analysed after spinal cord injury, 353 were men. Figure 2 shows the age distribution in decades. Figure 3 shows that the most frequent cause of injury was a traffic accident followed, by gunshot wounds, falls and diving accidents.

Of 106 patients who underwent neurosurgical treatment, 40 were operated on in the cervical spine with anterior discectomy and arthrodesis and 4 had a posterior approach with facetectomy and anterior arthrodesis; 6 others had a posterior arthrodesis only, at the $\mathrm{C} 1 / \mathrm{C} 2$ level. Twenty three patients were operated on for a thoracic spine injury; 16 only had a laminectomy and in 7 there was an associated arthrodesis using the Harrington-Luque Technique. Thirty six patients were operated on in the thoracolumbar region, 32 with HarringtonLuque stabilisation associated with a posterolateral decompression of the canal and 4 patients only had a fenestration.

Fifty four of the 105 patients were treated without an operation, and 51 underwent surgical treatment. Of those with a cervical spine injury, 15 were treated conservatively and 18 with surgery. In the thoracic region, 35 were treated conservatively and 20 with surgery, and in the lumbar region, 4 were treated conservatively and 13 with surgery. Table I analyses and details the surgical technique used.

\section{Result}

In those treated conservatively in Frankel Grade A (FG.A) the neurological deficits remained stable. In FG.B only $18.2 \%$ improved, and in those FG.C only $37.8 \%$ improved (Table II). 
Age

448 Patients

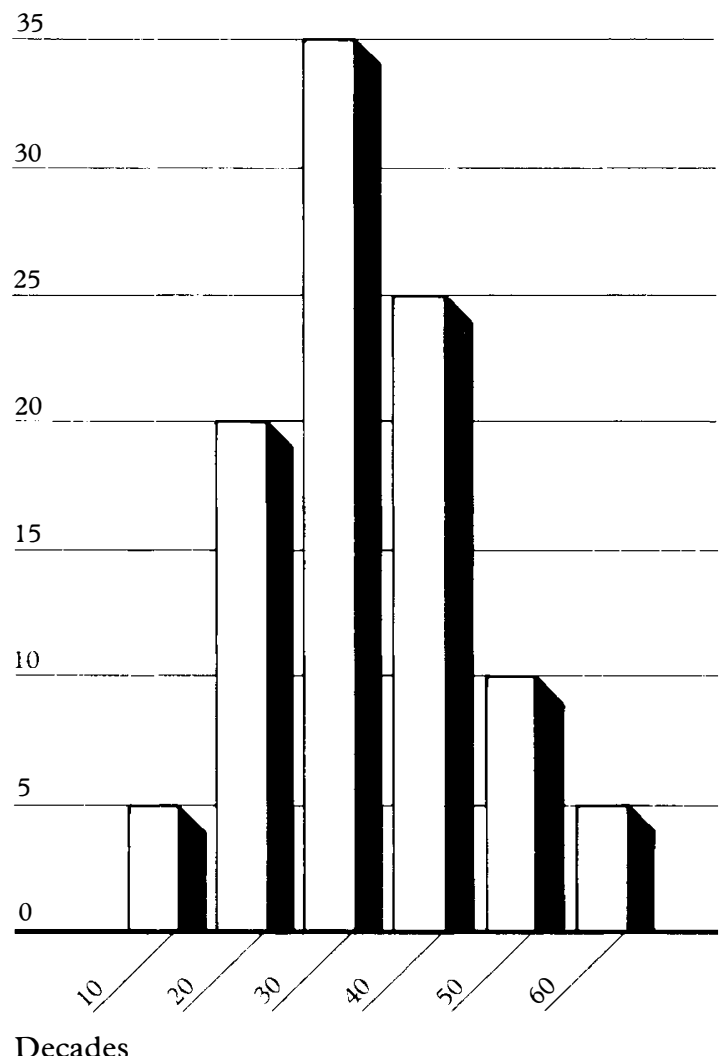

Figure 2 Age distribution.

Table I Type of surgical treatment

\begin{tabular}{lcccc}
\hline & Cervical & Dorsal & Lumbar & Total \\
\hline Anterior arthrodesis after discectomy (Cloward) & 13 & 00 & 00 & 13 \\
Laminectomies & 05 & 16 & 03 & 24 \\
Posterior Arthrodesis (Harrington-Luque) (only) & 00 & 03 & 08 & 11 \\
Laminectomy and posterior arthrodesis & 00 & 01 & 02 & 03 \\
Total & 18 & 20 & 13 & 51 \\
\hline
\end{tabular}

Regarding patients treated surgically, those in FG.A had no change in the neurological deficit. In FG.B $16.6 \%$ improved and those in FG.C 55.5\% improved (Table III). The outcome of the patients who did not have a spinal operation is set out in Table IV.

The independence in daily life activities for those with a cervical cord injury 
Cause and Regions Affected

No

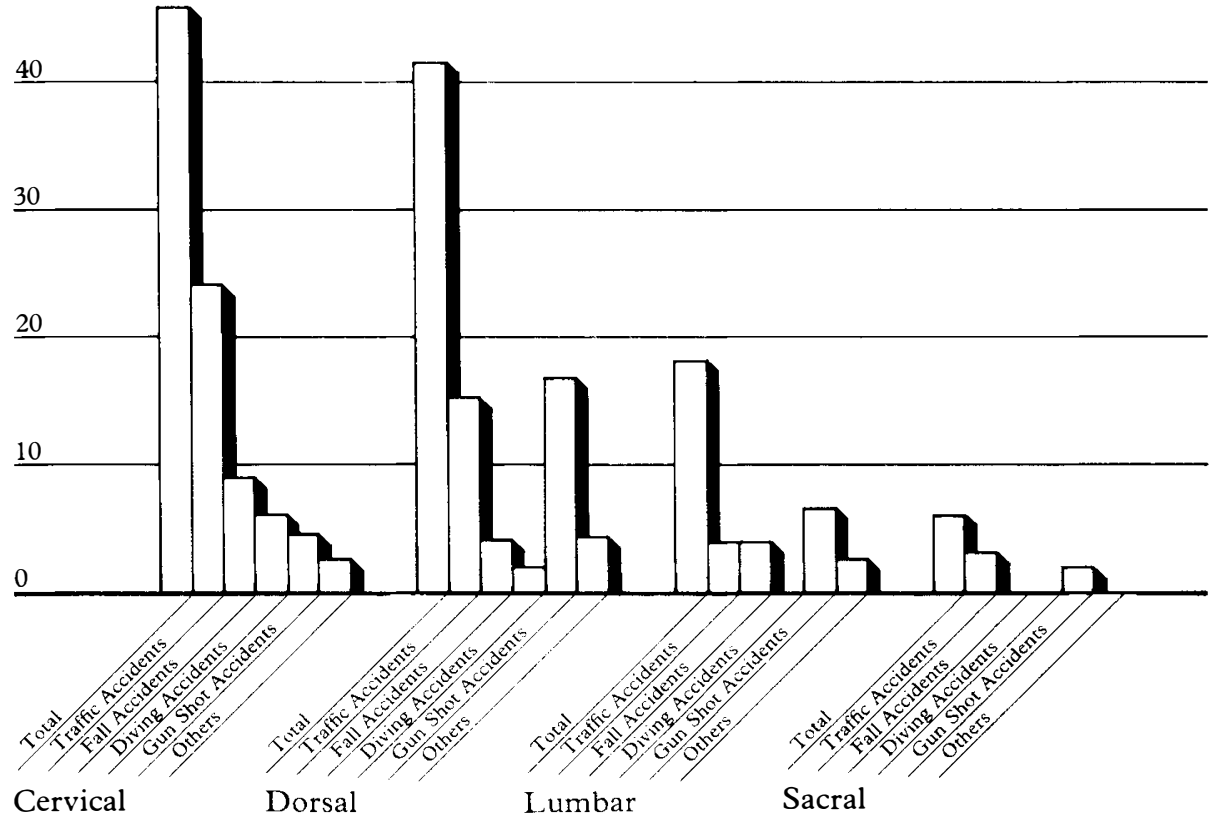

Figure 3 Aetiology and neurological level of injury.

Table II Result of non-surgical treatment (54 patients)

\begin{tabular}{ccccc}
\hline Grade & Total & Unchanged & Changed & Total \\
\hline A & 34 & 34 & 0 & 0 \\
B & 11 & 9 & 2 & $18.2 \%$ \\
C & 8 & 5 & 3 & $37.5 \%$ \\
D & 1 & 1 & 0 & 0 \\
\hline
\end{tabular}

Table III Result of surgical treatment (51 patients)

\begin{tabular}{ccccc}
\hline Grade & Total & Unchanged & Changed & Total \\
\hline A & 35 & 35 & 0 & 0 \\
B & 6 & 5 & 1 & $16.6 \%$ \\
C & 9 & 4 & 5 & $55.5 \%$ \\
D & 1 & 1 & 0 & 0 \\
\hline
\end{tabular}

Table IV Result of non-operative treatment (54 patients)

\begin{tabular}{ccccc}
\hline Grade & Total & Unchanged & Changed & Total \\
\hline A & 34 & 34 & 0 & 0 \\
B & 11 & 9 & 2 & $18.2 \%$ \\
C & 8 & 5 & 3 & $37.5 \%$ \\
D & 1 & 1 & 0 & 0 \\
\hline
\end{tabular}


Table $\mathbf{V}$ Dependency in daily life activity in cervical spinal lesions ( 32 patients)

\begin{tabular}{lcc}
\hline \multicolumn{1}{c}{ Daily life activity (DAL) } & Admission & Discharge \\
\hline Face hygiene & $91 \%$ & $31 \%$ \\
Body hygiene & $94 \%$ & $87 \%$ \\
Clothing & $97 \%$ & $90 \%$ \\
Feeding & $84 \%$ & $15 \%$ \\
Transfer & $97 \%$ & $90 \%$ \\
\hline
\end{tabular}

Table VI Dependency in daily life activity in thoracic and lumbar lesions (73 patients)

\begin{tabular}{lcc}
\hline \multicolumn{1}{c}{ Daily life activity (DAL) } & Admission & Discharge \\
\hline Face hygiene & $32 \%$ & 0 \\
Body hygiene & $67 \%$ & $6 \%$ \\
Clothing & $71 \%$ & $6 \%$ \\
Feeding & $29 \%$ & 0 \\
Transfer & $74 \%$ & $9 \%$ \\
\hline
\end{tabular}

is very low. An orthosis for the upper limbs to improve performance is necessary (Table V).

In the case of patients with thoracic and lumbar lesions, the aim was to reach total independency for daily life activities. This was achieved in 68 of 73 patients (Table VI).

For patients who could not walk, wheelchairs were necessary. The indication for braces was very limited.

Urinary bladder training was by intermittent catheterisation. On admission 50 patients had a Foley catheter, 9 were continent with suprapubic pressure, 39 were incontinent and 7 had normal control. At discharge 6 were still using a Foley catheter because of urinary problems. Forty five patients were continent with suprapubic pressure, 39 were incontinent, 15 had control and 2 used selfcatheterisation.

Bowel habits were initially developed using suppositories and laxatives at predetermined times. On admission 11 had control; 20 with habit and 74 without habit. On discharge 20 had control; 79 with habit and 6 without habit.

The most frequent complications occurring in spinal cord injury patients, are urinary tract infection and pressure sores. On admission a high percentage of patients had a urinary tract infection: $90 \%$ of the patients with a cervical lesion, $80 \%$ of those with a thoracic lesion, and $62 \%$ of those with a lumbar lesion. These percentages decreased respectively to $62 \%, 41 \%$ and $11 \%$ at the time of discharge. Analysing the pressure sores on admission, $53 \%$ of patients with a cervical lesion, $61 \%$ with a thoracic lesion and $40 \%$ with a lumbar lesion had pressure sores. During the hospital stay only $2 \%$ of the patients with a cervical lesion, and $1 \%$ of those with a thoracic lesion and none with lesion in the lumbar region developed pressure sores.

\section{Discussion}

The treatment of the injured spine remains controversial. There is some disagreement regarding indications and procedures, timing, the techniques, and 
the approach to be used. At first we believed that compression, edema and ischemia were the direct cause of the spinal cord lesion. Subsequently, after studies of the physiopathology, we saw that axial tensions and angulations were much more important factors. (Breig, 1960; Holdsworth, 1963; Kobrine, 1975; Torre, 1981). For these reasons, laminectomy has been relegated to a second place. An anterior approach to the cervical spine for decompression and stabilisation of the spinal column, and posterior decompression and stabilisation of the thoracolumbar column, are much more frequently indicated. There is some agreement in the literature. (Ducker, 1982; Erickson, 1983; Frankel et al., 1962; Holdsworth 1963 and Walters 1986). Comminuted fractures require surgical treatment to stop the progression of deformity. Patients with an incomplete neurological deficit, and a spinal cord compression in which conservative treatment has not decompressed the spinal cord and nerve roots, also require surgery.

There is some disagreement in the literature for surgery because of the high incidence of respiratory complications which may lead to death. Also some writers question the indication for surgery for the possibility of obtaining recovery of one or two nerve roots after adequate decompression and stabilisation of the spinal column.

Our protocol indicates surgery only for those patients with a good chance of recovery or where there is a risk of deterioration. After analysing our data we see that there is no change in the neurological deficit in patients with complete lesions after surgical or non-surgical treatment. In some of these patients it is necessary to stablise the spine externally or internally so that the patient can start rehabilitation as soon as possible. The level of the lesion did not seem to be important, but the grade of the lesion was fundamental regarding the prognosis. Those patients graded FG.C or FG.D should have treatment, conservatively, or surgically, because of the possibility to improve the neurological deficit. Patients with FG.B may have some improvement. The interventions are mandatory.

\section{Conclusion}

The goal of the treatment of the fractured spine is to restore and maintain the anatomical relationship between the spinal cord, nerve roots and the spinal canal, returning the patient as soon as possible to his functional capacity and social life. Surgical treatment seems to be indicated in those with comminuted fractures, unstable fractures, an incomplete spinal lesion with a persistent spinal canal deformity, and in those with a complete lesion, but with some possibility of the recovery of function of some nerve roots. It is worthwhile to stress that other objectives, not less important, are the maintenance of a good position in bed, personal care, good urinary function and mobile joints.

\section{References}

Breig A 1960 Biomechanics of the Central Nervous System. Alquimist and Wiksel, Stockholm. DuCKer TB, BellarriQue R 1982 Management of the fracture cervical spine. Cont. Neurosurg 4(16).

ERICKSON DL 1983 Clinical evaluation and surgical treatment of thoracolumbar fractures. Cont. Neurosurg 5(3). 
Frankel HL, Hancock DO, Hyslop G, et al. 1962 The value of postural reduction in the initial management of closed injuries of the spine with paraplegia and tetraplegia. Paraplegia 7:179-192.

Green AB, Callahan RA, Klose KJ, et al. 1981 Acute spinal cord injury: current concepts. Clinical Orthopaedics and Related Research 154:125-135.

HARRIS Jr JH 1978 Acute injuries of the spine. Seminars in Roentgenology 13(1):53-68.

HOLDSWORTH FW 1963 Fractures dislocations and fracture dislocations of the spine. fournal of Bone and foint Surgery 45B(1):6-20.

KoBRINE AK, MARTINS AN 1975 Local spinal cord blood flow in experimental traumatic myelopathy. Fournal of Neurosurgery 42:144.

Masini M, Freire Neto NG, Neves EGC, et al. 1986 Tratamento de fraturas toracolombares instáveis. Arquivos Brasileiros 5(3):143-154.

HAMILTON R 1979 Neurological programs after traumatic quadriplegia. fournal of Neurosurgery 50:611-666.

TORRE JC 1981 Spinal cord injury. Spine 6(4):315-335.

WALTERS CL, SCHMIDEK HH 1986 The management of thorocolumbar fractures. 8(20). 